By taking us beyond cure and disease control, Palliative Care continues where most medical and surgical texts end. There is a large measure of overlap with such texts in the early chapters on specific organ diseases. This detracts from much useful advice on symptom management. A third of the way through, however, the book begins to earn its cost. The chapters on the care of the dying child are both sensitive and practical. Those on the psychological aspects of pain and chronic diasbility show great insight. Advice on the management of pain and other symptoms is clearly and easily followed. Chapters on communication, bereavement and ethical issues are now standard in a book of this kind, but all three make worthwhile reading.

The foreword contains a description of how a patient was given the opportunity to give'. In spite of its cost, this book should help us in achieving that goal.

C. REGNARD,

The Churchill Hospital, Oxford OX3 $7 \mathrm{LJ}$.

\section{Saunders Dictionary and Encyclopedia of Laboratory Medicine and Technology}

Edited by James L. Bennington. Pp. xix +1674 , illustrated. W.

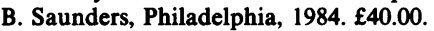

The laboratory worker of today needs to have a wide background knowledge of medical disorders, their appropriate investigation, the manner in which laboratory determinations are made and how their results should be interpreted. This volume embraces all these aspects of laboratory medicine. Clear, short descriptions of diseases, drugs, diagnostic investigations, techniques and interpretations are, included, and every aspect of pathology covered. Though less than a textbook, this book is far more than a dictionary. Encyclopedia is a more appropriate name to describe the great variety of topics and the mass of information carefully and accurately reported. This information is presented in readily accessible alphabetical order in a volume clearly printed and stoutly bound. It should be available to every clinical laboratory.

S. B. RosALKI, Royal Free Hospital, London NW3 2QG.
Shock Trauma/Critical Care Manual: Initial Assessment and Management

Edited by R. Adams Cowley and C. Michael Dunham. P xv +594 , illustrated. University Park Press, Baltimore (Distributed by Edward Arnold, Maidenhead), 1982. £38.00.

This manual of 594 pages is aimed at giving a "practical and clinici reference for the surgeon, the anaesthesiologist, the critical cafe specialist, the emergency room physician, the nurse and the house officer' in the management of the traumatized patient.

The book is based on the 20-year clinical evaluation of the shock trauma programme at the Maryland Institute of Emergency Medical Services Systems. Resuscitation at this Institute is conducted by orf of four trauma teams. Clearly, with such teams and a caseload much greater than the average Accident and Emergency (A. \& Ey Department in this country, it is possible to evolve a detaileg protocol to cater for all types of trauma.

The book is clearly laid out, broken into a logical sequence sections starting with initial evaluation and management, followed by the management of shock, systems management, and systern failure; and including a section on techniques and procedure monitoring, and a rather unexpected but excellent section on problems relating to the dying patient.

के

The text is brief and succinct, historical and pathophysiologich information is kept to a minimum; references are absent. Dispersed between practical aspects of management are relevant and perceptive inserts entitled 'Perspectives'-giving a brief and rational explanation of the procedures described. More use could have been made of diagrams to illustrate management sequence or proceduref.

The book is excellently compiled. It might be of value in a busy $\mathbf{A}$. \& E. Department for ready reference by house officers, but because of differing techniques and the greater variety of drugs available this country, could not be followed step-by-step for patient management. In fact, one of the few omissions in the text is deten on drug therapy which may be used during patient management. 0

In summary, this book might prove valuable as a basio fog teaching and discussion for junior staff and nurses in $A$. departments regarding management of the traumatized patient. should also encourage busy A. \& E. departments in this country develop protocols of similar layout and clarity.

Gillian C. Hanson, Whipps Cross Hospital, 응 London E11 INR. 